\title{
In Vivo Vascular Casting
}

\author{
J. F. Hainfeld*, D. N. Slatkin*, T. M. Focella* and H. M. Smilowitz** \\ * Nanoprobes, Inc., 95 Horse Block Road, Yaphank, NY, 11980 \\ **University of Connecticut Health Center, Farmington, CT 06030
}

The vascular casting technique has permitted highly illuminating visualization of the blood supply to various tissues and organs. Unfortunately it currently requires polymerization of the cast material within the animal and is a terminal procedure. Therefore, interesting in vivo processes cannot be monitored in the same animal over time to see real-time changes occurring normally or in response to the environment, diet, drugs, infection, tumor growth and many other interesting biological processes.

Here we introduce a method to overcome this limitation by development of highly tolerated gold nanoparticles that provide enough contrast in the vascular system to reconstruct its architecture to high resolution using X-rays. Gold nanoparticles, $1.9 \mathrm{~nm}$ in diameter (Fig. 1), were synthesized and found to be highly tolerated in test mice. After $2.7 \mathrm{~g} \mathrm{Au} / \mathrm{kg}$, injected iv, mice lived over one year without any signs of illness [1]. This produced an initial blood concentration of $36 \mathrm{mg} \mathrm{Au} / \mathrm{ml}$ (i.e., blood was 3.6\% gold by weight). With such a high level, the blood vessels were clearly seen using a mammography unit (Fig. 2). Vessels as small as 100 microns in diameter could be discerned. Vascular casting may then be accomplished by using a CT or microCT. Resolution limits of microCT machines is typically about 10 microns, but of course motion of the animal must be contended with as well as the time needed to acquire such data, since scan times are much slower than for human CT scanners.

Post-mortum histology may also be used to further examine the distribution of the gold nanoparticles. An interesting tool is silver or gold enhancement that specifically visualizes the tiny gold nanoparticles and can show their intracellular and extracellular biodistribution. An example is shown in Fig. 3 for kidney sections.

\section{References}

[1] J. Hainfeld et al., Phys. Med. Biol. 49 (2004) N309. 


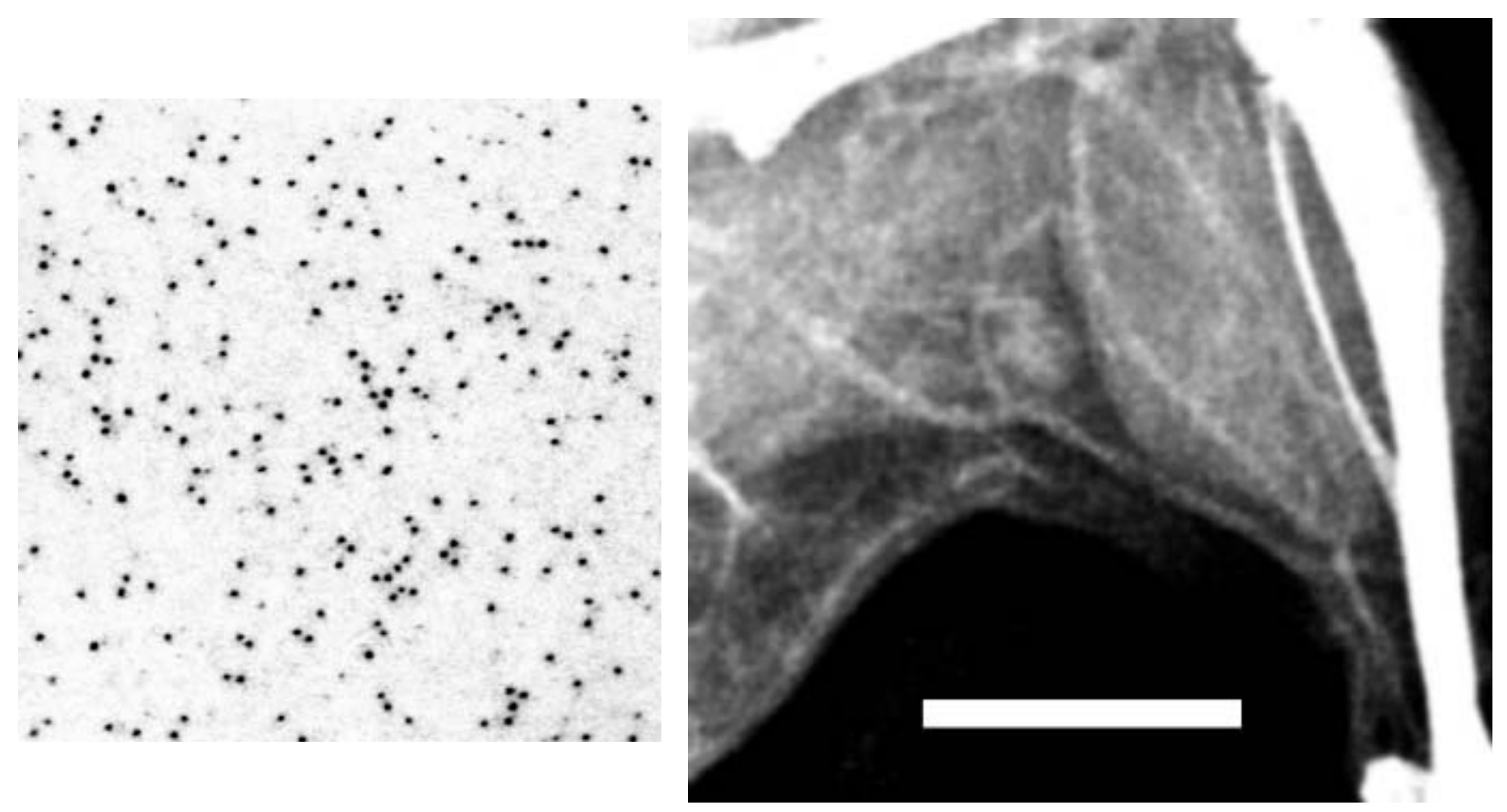

Fig. 1 (Left): EM image of $1.9 \mathrm{~nm}$ gold nanoparticles (full width $130 \mathrm{~nm}$ ).

Fig. 2 (Right): X-ray radiograph of the hindleg of a live mouse after iv injection of gold nanoparticles. Bar $=5 \mathrm{~mm}$.
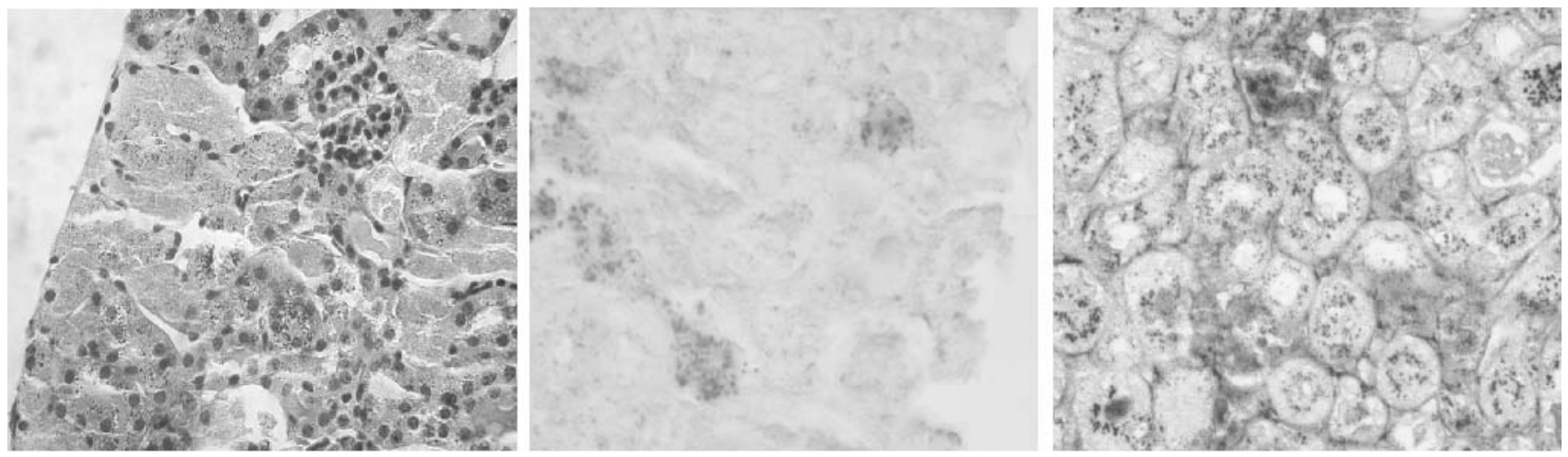

Fig. 3. Left is H\&E of mouse kidney after a large iv injection of $1.9 \mathrm{~nm}$ gold particles, Middle is unstained kidney (same block) showing large Au accumulations, and Right is after autometallographic enhancement showing where the small gold particles were localized (no other stain used); 400x. 